\title{
Uncertainty Quantification in Remaining Useful Life of Aerospace Components using State Space Models and Inverse FORM
}

\author{
Shankar Sankararaman* \\ SGT Inc., NASA Ames Research Center, Moffett Field, CA 94035, USA \\ Kai Goebel ${ }^{\dagger}$ \\ NASA Ames Research Center, Moffett Field, CA 94035, USA
}

\begin{abstract}
This paper investigates the use of the inverse first-order reliability method (inverseFORM) to quantify the uncertainty in the remaining useful life (RUL) of aerospace components. The prediction of remaining useful life is an integral part of system health prognosis, and directly helps in online health monitoring and decision-making. However, the prediction of remaining useful life is affected by several sources of uncertainty, and therefore it is necessary to quantify the uncertainty in the remaining useful life prediction. While system parameter uncertainty and physical variability can be easily included in inverse-FORM, this paper extends the methodology to include: (1) future loading uncertainty, (2) process noise; and (3) uncertainty in the state estimate. The inverse-FORM method has been used in this paper to (1) quickly obtain probability bounds on the remaining useful life prediction; and (2) calculate the entire probability distribution of remaining useful life prediction, and the results are verified against Monte Carlo sampling. The proposed methodology is illustrated using a numerical example.
\end{abstract}

\section{Introduction}

The use of accurate system health management tools is indispensable for safety-critical and missioncritical aerospace systems. It is important to constantly monitor the performance of these systems, identify faults (diagnosis), predict possible failures in the near future, and quantify the remaining useful life (prognosis) in order to aid online decision-making. Sometimes, it may be challenging to perform health monitoring on the whole system due to its sheer complexity, and therefore, diagnosis and prognosis need to performed on individual components which constitute the overall system. In this approach, mathematical models are developed for individual components, and then the component models are integrated to form the overall system. These models can be used in the health monitoring to guide in model-based diagnostics ${ }^{11}$ and prognostics. $^{2}$ Alternatively, data-driven approaches ${ }^{3}$ are also available for health monitoring, but they are not discussed in the present paper.

Uncertainty quantification and management are important aspects of health monitoring, especially due to the presence of several unknown factors which affect the performance of the system of interest. It is not only important to develop robust algorithms for diagnosis and prognosis, i.e., accurately perform diagnosis and prognosis in the presence of uncertainty, but also important to quantify the amount of confidence in the results of diagnosis and prognosis to enable in-flight decision-making capabilities. Sankararaman and Mahadevar 4 [5 developed statistical (both frequentist and Bayesian) approaches to quantify the uncertainty in the three steps of diagnosis (detection, isolation, quantification/estimation) in an online health monitoring framework. There have also been a few papers $\frac{6}{6]}[8 \mid 9]$ which discuss uncertainty propagation in prognosis; however, many of these papers are either suitable only for offline prognosis or they do not provide a comprehensive treatment of uncertainty ${ }^{[6]}$ Further research is necessary to develop rigorous uncertainty quantification methods which

${ }^{*}$ Post-doctoral Research Scholar, NASA Ames Research Center, Moffett Field, CA 94035, USA.

${ }^{\dagger}$ Intelligent Systems Division, NASA Ames Research Center, Moffett Field, CA 94035, USA 
are suitable for online prognostics and decision-making. The rest of the paper focuses on one particular aspect of prognostics, i.e., quantifying the uncertainty in the remaining useful life (RUL) prediction. Since state space equations are suitable for representing time-dependent behavior, the governing differential equations of the component (of the system of interest) are transformed into equivalent state space representations that collectively serve as the component model for performance prediction and RUL computation. The first and foremost challenge with respect to the estimation of RUL uncertainty is the characterization of uncertainty in the future loading. Though a few publications have addressed in this issue, $\frac{10}{11}$ further research is needed in this direction. However, the focus of the present paper is not on uncertainty characterization, and therefore it is assumed that future loading variability has been characterized based on already existing loading profiles and data sets. The other sources of uncertainty include the process noise of the state space model (a component of modeling error), the uncertainty in the current state estimates, uncertainty in the model parameters, etc. Note that state estimation is a prerequisite to prognosis, and a filtering algorithm is used along with measurement data to estimate the current state. The data uncertainty associated with these measurements directly contributes to the estimated uncertainty in the component states.

Conventionally, prognosis has been performed using sampling-based approaches (for example, particle filtering without the correction-step); since such methods may require several samples or particles in order to predict the RUL, and may not be suitable for online decision-making. Moreover, sampling-based based algorithms are "non-deterministic"; for example, every instance of a particle filter may lead to a different probability distribution for the RUL. On the other analytical methods such as the first-order reliability method (FORM), though approximate, can overcome both of these challenges. In particular, the inverse first-order reliability method (inverse-FORM) can be used to calculate probability bounds on RUL (in fact, the entire probability distribution of RUL at discrete probability levels), which are useful for decisionmaking. Though such analytical methods have been used for time-dependent reliability calculation, $12[13$ their application to state space models poses several new challenges. The primary contribution of this paper is to extend the inverse FORM approach to include state space models and compute the uncertainty in the RUL prediction. The inverse-FORM approach not only requires fewer prognostic evaluations in comparison with the sampling-based approaches but can also produce repeatable calculations, i.e., the exact same probability distribution on every repetition of the algorithm. While the former directly aids in online prognosis since fewer evaluations would lead to quicker calculations, it is worth noting that the latter feature is an important criterion for existing verification, validation, and certification protocols in the aerospace domain. Therefore, investigating such analytical algorithms allows us to move a step closer towards adopting prediction algorithms, by meeting the needs of the current certification process. The rest of this paper is organized as follows. Section $\Pi$ discusses the various challenges involved with using inverse FORM along with state space models, and develops computational methods to address them so that the uncertainty in the RUL prediction can be computed. Section III illustrates the proposed methods using a numerical example and conclusions are presented in Section [V]

\section{Quantifying Uncertainty in RUL Prediction}

First, the inverse-FORM approach is briefly explained in subsection $\Pi \mathrm{A}$. In subsection $\Pi \mathrm{B}$, a generalized framework for the computation of RUL is described, and then, the challenges of using FORM with such statespace models is explained in subsection [IIC] The proposed method is described in subsection IIID, and finally, the inverse-FORM algorithm is summarized in subsection $\amalg \mathrm{E}$.

\section{A. The Inverse FORM Approach}

Consider a generic computational model $Y=H(\boldsymbol{X})$, which is used to represent the performance of an engineering system. The input is a vector and hence denoted in bold as $\boldsymbol{X}$, whereas the output $Y$ is a scalar. The model $G$ is deterministic, i.e. for a given realization of $\boldsymbol{X}$, there is a corresponding output, which is a realization of $Y$. The inputs $\boldsymbol{X}$ are uncertain, and this leads to uncertainty in the output $Y$. A generic realization of $\boldsymbol{X}$ is denoted as $\boldsymbol{x}$, and a generic realization of $Y$ is denoted as $y$. Given the probability distributions of $\boldsymbol{X}$, FORM and inverse-FORM are tools for uncertainty propagation, i.e., to calculate the cumulative distribution (CDF, denoted by $F_{Y}(y)$ ) or the probability density function (PDF, denoted by $\left.f_{Y}(y)\right)$.

The focus of inverse-FORM ${ }^{14}$ is to select the value of $y_{c}$ such that the cumulative distribution function 


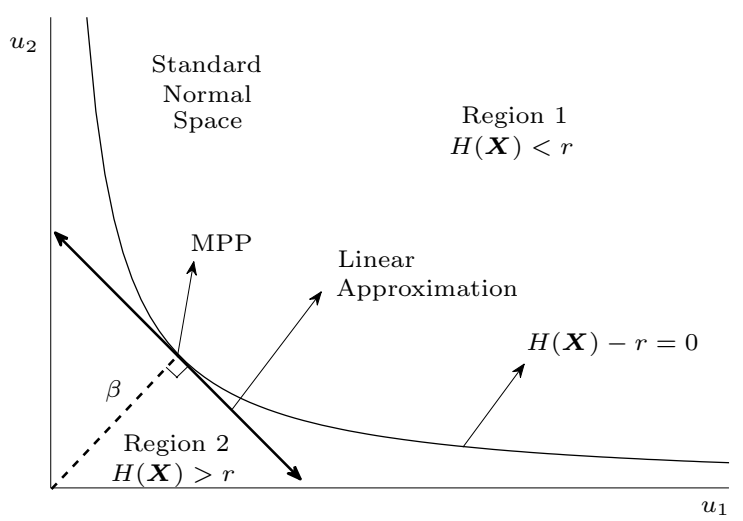

Figure 1: Estimating MPP in inverse-FORM

$(\mathrm{CDF})$ value measured at $y_{c}$ is equal to a given probability value $\alpha$, i.e. select $y_{c}$ such that $F_{Y}\left(Y=y_{c}\right)=\alpha$. The basic concept is to "linearize" the model $H$ so that the the output $Y$ can be expressed as a linear combination of the random variables. First, all the random variables are transformed into uncorrelated standard normal space; in this transformed space, the point of linearization is chosen such that it is closest to the origin, implying that it has the highest probability of occurrence (Most Probable Point or MPP). Further, in the transformed space, the output $Y$ is also a normal variable because a linear combination of normal variables is normal. Therefore, the CDF value $F_{Y}\left(Y=y_{c}\right)$ can be computed using the standard normal distribution function in terms of the minimum distance (denoted by $\beta$ in Fig. 1) as $F_{Y}\left(Y=y_{c}\right)=\Phi(-\beta)$. The transformation of random variables $\boldsymbol{X}$ into uncorrelated standard normal space $(\boldsymbol{U})$ is denoted by $\boldsymbol{U}=T(\boldsymbol{X})$, and the details of the transformation can be found in Haldar and Mahadevan $\frac{15}{15}$ An iterative procedure (in each iteration, (1) the transformation is repeated; and (2) the point of "linearization" changes when the " $H$ " is non-linear and $\boldsymbol{X}$ are non-normal variables) for inverse-FORM can be found several publications (for e.g., Li and Foschi ${ }^{16}$ ). The iterative procedure starts at an arbitrary $\boldsymbol{x}$ and continues until all the optimality conditions are satisfied.

While inverse-FORM has commonly been used to calculate $y_{c}$ corresponding to a given $\alpha$, it can also be used to compute the entire CDF by repeating the inverse-FORM analysis for multiple values of $\alpha$ (different probability levels). For example, if inverse-FORM is performed at 10 different values of $\alpha$, the corresponding $y_{c}$ values are calculated, and an interpolation scheme can be used to calculate the entire CDF (which can be differentiated to obtain the PDF).

\section{B. Generalized State Space Model for RUL Prediction}

State space models are generally suitable to represent time-dependent behavior, and therefore, such models are commonly used for prognosis and RUL prediction. A general state space model can be represented as:

$$
\dot{\mathbf{x}}(t)=\mathbf{f}(t, \mathbf{x}(t), \boldsymbol{\theta}(t), \mathbf{u}(t), \mathbf{v}(t))
$$

where $\mathbf{x}(t) \in \mathbb{R}^{n_{x}}$ is the state vector, $\boldsymbol{\theta}(t) \in \mathbb{R}^{n_{\theta}}$ is the parameter vector, $\mathbf{u}(t) \in \mathbb{R}^{n_{u}}$ is the input vector, $\mathbf{v}(t) \in \mathbb{R}^{n_{v}}$ is the process noise vector, $\mathbf{f}$ is the state equation.

The goal of prognostics is to predict the remaining useful life (RUL) at any generic prediction time $\left(t_{P}\right)$. The first step is to estimate the state at this prediction time, i.e., $\mathbf{x}\left(t_{P}\right)$; such estimation is typically performed using filtering approaches (particle filtering, Kalman filtering) using the data available until the prediction time $\left(t_{P}\right)$. Let $\mathbf{y}(t) \in \mathbb{R}^{n_{y}}, \mathbf{n}(t) \in \mathbb{R}^{n_{n}}$, and $\mathbf{h}$ denote the output vector, measurement noise vector, and output equation respectively. Then,,

$$
\mathbf{y}(t)=\mathbf{h}(t, \mathbf{x}(t), \boldsymbol{\theta}(t), \mathbf{u}(t), \mathbf{n}(t))
$$

Note that the output equation or data is not used in the prognosis stage, since the focus is on predicting the future and the associated uncertainty. 
Prognostics and RUL prediction is concerned with the performance of the component that lies outside a given region of acceptable behavior. The desired performance is expressed through a set of $n_{c}$ constraints, $C_{E O L}=\left\{c_{i}\right\}_{i=1}^{n_{c}}$, where $c_{i}: \mathbb{R}^{n_{x}} \times \mathbb{R}^{n_{\theta}} \times \mathbb{R}^{n_{u}} \rightarrow \mathbb{B}$ maps a given point in the joint state-parameter space given the current inputs, $(\mathbf{x}(t), \boldsymbol{\theta}(t), \mathbf{u}(t))$, to the Boolean domain $\mathbb{B} \triangleq[0,1]$, where $c_{i}(\mathbf{x}(t), \boldsymbol{\theta}(t), \mathbf{u}(t))=1$ if the constraint is satisfied, and 0 otherwise.

These individual constraints may be combined into a single threshold function $T_{E O L}: \mathbb{R}^{n_{x}} \times \mathbb{R}^{n_{\theta}} \times \mathbb{R}^{n_{u}} \rightarrow$ $\mathbb{B}$, defined as:

$$
T_{E O L}(\mathbf{x}(t), \boldsymbol{\theta}(t), \mathbf{u}(t))= \begin{cases}1, & 0 \in\left\{c_{i}(\mathbf{x}(t), \boldsymbol{\theta}(t), \mathbf{u}(t))\right\}_{i=1}^{n_{c}} \\ 0, & \text { otherwise. }\end{cases}
$$

$T_{E O L}$ is equal to 1 when any of the constraints are violated. Then, the End of Life (EOL, denoted by $E$ ) at any time instant $t_{P}$ is then defined as the earliest time point at which this occurs:

$$
E\left(t_{P}\right) \triangleq \inf \left\{t \in \mathbb{R}: t \geq t_{P} \wedge T_{E O L}(\mathbf{x}(t), \boldsymbol{\theta}(t), \mathbf{u}(t))=1\right\}
$$

The Remaining Useful Life (RUL, denoted by $R$ ) at time instant $t_{P}$ is expressed as:

$$
R\left(t_{P}\right) \triangleq E\left(t_{P}\right)-t_{P}
$$

Thus, it is clear that $R\left(t_{P}\right)$ depends on (1) present time $\left(t_{P}\right)$; (2) present state estimate $\left(\mathbf{x}\left(t_{P}\right)\right)$; (3) future loading $\left(\mathbf{u}\left(t_{P}: t_{P}+R\left(t_{P}\right)\right)\right) ;(4)$ parameters $\left(\boldsymbol{\theta}\left(t_{P}: t_{P}+R\left(t_{P}\right)\right)\right)$; and (5) process noise $\left(\mathbf{v}\left(t_{P}: t_{P}+R\left(t_{P}\right)\right)\right)$. Since these quantities may be uncertain, RUL also becomes uncertain, and as described earlier, the goal of this paper is to quantify this uncertainty. For the sake of illustration, assume that the parameters are known deterministically; even if they are not, parameter uncertainty can be treated to the other sources of uncertainty.

Note: The symbol " $\mathbf{u}\left(t_{P}: t_{P}+R\left(t_{P}\right)\right)$ " refers to the entire loading from time " $t=t_{P}$ " until time " $t=t_{P}+R\left(t_{P}\right)$ ". Similarly, the parameter values and process noise values during this time period can be written as " $\boldsymbol{\theta}\left(t_{P}: t_{P}+R\left(t_{P}\right)\right)$ " and "v $\left(t_{P}: t_{P}+R\left(t_{P}\right)\right)$ " respectively..

\section{Challenges}

The first challenge is that the dependence of $R\left(t_{P}\right)$ on other uncertain quantities is implicit, i.e., the RUL depends on the process noise and loading, and in turn, "how long the process noise and the future loading need to be considered during prognosis" directly depends on the RUL itself.

In the past, 17 time-variant random loading has often be decomposed into two parts: (1) time-variant deterministic component; and (2) time-invariant random component. Therefore, $\left.\mathbf{u}\left(t_{P}: t_{P}+R\left(t_{P}\right)\right)\right)$ can be replaced with $\mathbf{u}^{E}$ (the equivalent, time-invariant random components), and thereby, such decomposition facilitates the treatment of future loading uncertainty. However, the treatment of process noise (usually, but not necessarily, considered to be normally distributed, i.e., $\left.\mathbf{v}(t) \sim N\left(0, \Sigma_{t}^{2}\right)\right)$ is not trivial, because $\mathbf{v}\left(t_{1}\right)$ and $\mathbf{v}\left(t_{2}\right)$ need to be treated as two different random quantities (there may be correlation across time, which is not considered in this paper). So, there are " $R\left(t_{P}\right) \times$ time-discretization" number of process noise terms; note the presence of implicitness since this is again a function of the RUL itself.

The next challenge is to identify the function " $H$ " discussed earlier in Section $\mathrm{A}$ The obvious choice is to use the state evolution equation, i.e, Eq. 1) as " $H$ ". Then, this would mean performing uncertainty quantification across every time-step, thereby predicting the entire probability distribution of all the state variables $\left(\mathbf{x}\left(t_{P}: t_{P}+R\left(t_{P}\right)\right)\right)$ continuously as a function of time. However, this is a cumbersome process because of the fact that there may be several states which are highly statistically dependent (sometimes, they may be even functionally dependent), and therefore, it is important to retain the correlation information all along. Further, these state variables may not be normally distributed, and every inverse-FORM analysis would require a transform from correlated non-normal space to uncorrelated standard normal space, thus complicating the procedure. In the next subsection, a statistical method is proposed in order to overcome these challenges and facilitate the implementation of inverse-FORM for RUL calculation.

\section{Proposed Method}

Since the focus of this paper is to compute the uncertainty in the RUL, it is proposed that a new deterministic transfer function " $G$ " be constructed, so that the inputs are realizations of uncertain quantities, and the 
output is the corresponding realization of RUL. This function can be written as:

$$
R\left(t_{P}\right)=G\left(\mathbf{x}\left(t_{P}\right), \mathbf{u}^{E}, \mathbf{v}\left(t_{P}: t_{P}+R\left(t_{P}\right)\right)\right)
$$

This function can be evaluated by continuously solving Eq. 1 until failure occurs (when $T_{E O L}$ changes the value from 0 to 1 ). While this " $G$ " is similar to " $H$ " in Sec A there are two more challenges that need to be addressed. First, the above equation is still implicit, because the number of process noise terms is dependent on $R\left(t_{P}\right)$. Second, if $R\left(t_{P}\right)$ is discretized into several hundreds of steps, then there are several hundreds of inputs to " $G$ ". This poses a computational challenge to inverse-FORM because of the need to compute the gradient that may now have several hundreds of terms. Therefore, to obtain the entire PDF (repeating inverse-FORM for 10 different probability levels), several thousands of evaluations of " $G$ " may be necessary. Therefore, this approach becomes more expensive than sampling methods, and not suitable for online health monitoring.

This paper proposes a new likelihood-based method to overcome this challenge. Suppose that the same realization of process noise $\mathbf{v}(t)=\mathbf{v}^{E}$ is considered for all time-steps $t \geq t_{P}$, i.e., the process noise is timeinvariant (constant at all time steps after prediction time $t_{P}$ ). Then, the likelihood for the occurrence of this event can be written as:

$$
L\left(\mathbf{v}^{E} \mid R\left(t_{P}\right)\right) \propto \prod_{t=t_{P}}^{t=t_{P}+R\left(t_{P}\right)} f_{\mathbf{V}(t)}\left(\mathbf{V}(t)=\mathbf{v}^{E}\right)
$$

where $f_{\mathbf{V}(t)}(\mathbf{v}(t))$ is the probability density function of $\mathbf{V}(t)$. Also note that the likelihood function is conditioned on the RUL and written as $R\left(t_{P}\right)$. Further, the above equation assumes that the process noise values at two different times are independent of each other. If any statistical dependence is unknown, then it can be easily included in the above equation by conditioning appropriately. Having calculate the likelihood of this event, the PDF of $\mathbf{v}^{E}$ can be calculated as: $\frac{18}{18}$

$$
f_{\mathbf{V}^{E}}\left(\mathbf{v}^{E} \mid R\left(t_{P}\right)\right)=\frac{L\left(\mathbf{v}^{E} \mid R\left(t_{P}\right)\right)}{\int_{D} L\left(\mathbf{v}^{E} \mid R\left(t_{P}\right)\right) d \mathbf{v}^{E}}
$$

In Eq. 8. the domain of integration $D$ is chosen such that $\mathbf{v}^{E} \in D$ if and only if $L\left(\mathbf{v}^{E}\right) \neq 0$.

Now, suppose that, in Eq. 6] the time-variant process is replaced with a time-invariant constant. In other words, $\left.\mathbf{v}\left(t_{P}: t_{P}+R\left(t_{P}\right)\right)\right)$ is replaced with $\mathbf{v}^{E}$, as:

$$
R\left(t_{P}\right)=G\left(\mathbf{x}\left(t_{P}\right), \mathbf{u}^{E}, \mathbf{v}^{E}\right)
$$

The above discussion means that a suitable probability distribution needs to be chosen for $\mathbf{v}^{E}$ so that the effect of propagating this distribution through Eq. 9 is equivalent to propagating the original distribution of $\mathbf{V}(t)$ through Eq 6. In fact, Eq. 7 and 8 accomplish this goal by matching corresponding probability densities. Hereon, $\mathbf{v}^{E}$ is referred to as equivalent time-invariant process noise and the distribution corresponding to the PDF $f_{\mathbf{V}^{E}}\left(\mathbf{v}^{E} \mid R\left(t_{P}\right)\right)$ is referred to as the equivalent invariant process noise distribution.

Now the function " $G$ " is not implicit, because $\mathbf{v}^{E}$ is simply a time-invariant constant and $R\left(t_{P}\right)$ does not appear on the right hand side of Eq. 9. On the other hand, the PDF of $\mathbf{v}^{E}$ is conditionally dependent on $R\left(t_{P}\right)$, and this dependence can be expressed explicitly. In other words, for a given realization of $\mathbf{v}^{E}$, Eq. 9 can be used to compute $R\left(t_{P}\right)$, which can then be used to calculate the PDF $f_{\mathbf{V}^{E}}\left(\mathbf{v}^{E} \mid R\left(t_{P}\right)\right)$. Hence, any iterative algorithm for inverse-FORM can now be applied for RUL estimation by performing one additional step, i.e., calculating the probability density function of the current value of $\mathbf{v}^{E}$, which is then used to transforming the variables to the standard normal space.

\section{E. Algorithm: Summary}

The section summarizes the algorithm used in this paper and explains how inverse-FORM is to compute the value of RUL that corresponds to a given probability level, i.e., compute $r$ for a given $\alpha$ such that $F_{R}(r)=\alpha$. To begin with, calculate $\beta$ such that $\alpha=P h i(-\beta)$, and use the following algorithm to calculate $r$.

1. Start with an initial guess of all the quantities which RUL depends on, as in Eq. 9. Initialize $j=0$, and let the vector of initial guesses be denoted by $\boldsymbol{x}^{j}$. 
2. Transform $\boldsymbol{x}^{j}$ into standard normal space $\boldsymbol{u}^{j}$, using any of the commonly used transforms. $\frac{15}{15}$ The probability distribution of $v^{E}$ needs to be calculated as in Eq. 8 . Note that the value of $R\left(t_{P}\right)$ is also calculated for each iteration and this facilitates the computation in Eq. 8 .

3. Calculate the gradient $(\alpha)$ in the standard normal space, and update the location using the gradient, i.e., $\boldsymbol{u}^{j+1}=\frac{\alpha}{|\alpha|} \times \beta$.

4. Re-transform into original space using the inverse of the previously used standard normal transformation, and obtain $\boldsymbol{x}^{j+1}$.

5. Repeat steps 2-4 until convergence, i.e., $\boldsymbol{x}^{j}=\boldsymbol{x}^{j+1}$ and $\left|\boldsymbol{x}^{j}\right|=\beta$.

The following section presents a numerical example to illustrate the above algorithm; first the state-space model is discussed in detail, and then the proposed method is used to quantify the uncertainty in remaining useful life prediction.

\section{Numerical Example}

The numerical example consists of a lithium-ion battery, which is an important component of the rover test-bed $\frac{19}{19}$ being studied at NASA Ames Research Center. Though the method is illustrated using a battery model, it is general enough to be applicable to state space models in several engineering domains.

\section{A. Description of the Model}

The battery model is based on an electrical circuit equivalent shown in Fig. 2, similar to models presented by Chen and Rincon-Mora ${ }^{20}$ The large capacitance $C_{b}$ holds the charge $q_{b}$ of the battery. The $R_{C P}-C_{C P}$ pair captures the major nonlinear voltage drop due to concentration polarization, $R_{s}$ captures the so-called I-R drop, and $R_{p}$ models the parasitic resistance that accounts for self-discharge. This simple battery mode ${ }^{21] 22}$ is enough to capture the major dynamics of the battery, but ignores temperature effects and other minor battery processes.

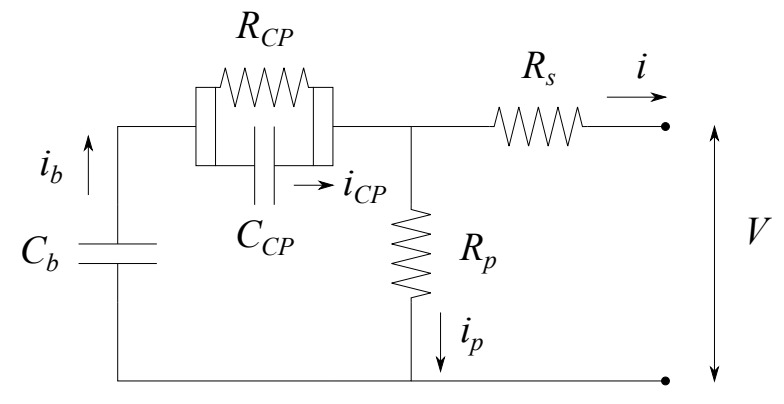

Figure 2: Battery equivalent circuit

The state-of-charge, $S O C$, is computed as

$$
S O C=1-\frac{q_{\max }-q_{b}}{C_{\max }}
$$

where $q_{b}$ is the current charge in the battery (related to $C_{b}$ ), $q_{\max }$ is the maximum possible charge, and $C_{\max }$ is the maximum possible battery capacity (i.e., nominally, its rated capacity). The concentration polarization resistance is a nonlinear function of $S O C$ :

$$
R_{C P}=R_{C P 0}+R_{C P 1} \exp \left(R_{C P 2}(1-S O C)\right)
$$

where $R_{C P 0}, R_{C P 1}$, and $R_{C P 1}$ are empirical parameters. The resistance, and, hence, the voltage drop, increases exponentially as $S O C$ decreases $\frac{10}{10}$ 
Voltage drops across the individual circuit elements are given by

$$
\begin{aligned}
V_{b} & =q_{b} / C_{b} \\
V_{C P} & =q_{C P} / C_{C P} \\
V_{p} & =V_{b}-V_{C P}
\end{aligned}
$$

where $q_{C P}$ is the charge associated with the capacitance $C_{C P}$. The terminal voltage of the battery is

$$
V=V_{b}-V_{C P}-R_{s} i
$$

where $i$ is the battery current at the terminals. Currents associated with the individual circuit elements are given by

$$
\begin{aligned}
i_{p} & =V_{p} / R_{p} \\
i_{b} & =i_{p}+i \\
i_{C P} & =i_{b}-V_{C P} / R_{C P}
\end{aligned}
$$

The charges are then governed by

$$
\begin{aligned}
\dot{q}_{b} & =-i_{b} \\
\dot{q}_{C P} & =i_{C P}
\end{aligned}
$$

It is of interest to predict the end-of-discharge as defined by a voltage threshold $V_{E O D}$. So, $C_{E O L}$ consists of only one constraint:

$$
c_{1}: V>V_{E O D}
$$

The parameters of the battery model are assumed to be deterministic and are shown in Table 1

Table 1: Battery Model Parameters

\begin{tabular}{c|c}
\hline Parameter & Value \\
\hline$C_{b}$ & 9844 \\
$R_{s}$ & 0.143014 \\
$R_{p}$ & 500 \\
$C_{C P}$ & 70.3767 \\
$R_{C P 0}$ & 0.019829 \\
$R_{C P 1}$ & $3.68606 \times 10^{-14}$ \\
$R_{C P 2}$ & 31.9213 \\
$q_{\max }$ & 41400 \\
$C_{\max }$ & 6900 \\
\hline
\end{tabular}

\section{B. Verification of the Equivalent Time-invariant Approach}

Before quantifying the uncertainty in remaining useful life (RUL) prediction, this section focuses on verifying the proposed equivalent time-invariant approach, by suppressing all the other sources of uncertainty except process noise. Since there are two state variables in this example, two process noise terms are present. The two initial states are assumed to $\left[41.4 \times 10^{-3}, 0\right]$, and time-invariant PDFs are chosen for the two process noise terms with normal distributions of $N(0,1)$ and $N(0,0.1)$ respectively; these are the probability distributions at any time instant and used to calculate the probability distributions in Eq 8 . Even though small values of standard deviation are chosen, they have a significant impact on the RUL distribution, as shown below.

The statistical correctness of the proposed equivalent time-invariant approach is independent of the uncertainty propagation technique (sampling-based or analytical) used. Therefore, for the sake of rigorous verification, Monte Carlo sampling (MCS) is used to compare the CDFs obtained by using the original process noise distribution and the equivalent invariant process noise distribution, i.e.,

1. Proposed time-invariant approach, i.e. same value of process noise at all time steps, as in Eq. 9. The resultant $\mathrm{CDF}$ is indicated as "Invariant Noise" in Fig. 3. 


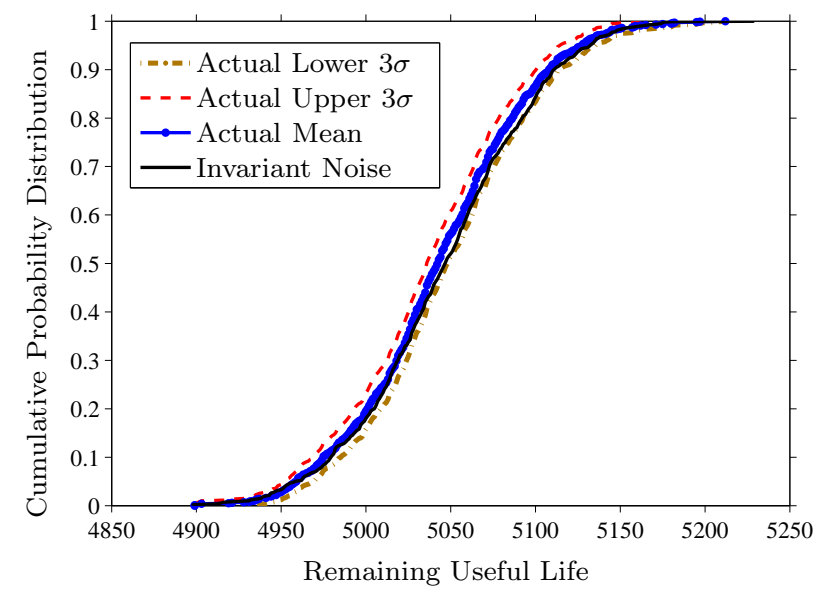

Figure 3: Verification of the Proposed Equivalent Time Invariant Approach

2. Actual process noise, i.e., sampling independent realizations of process noise in each and every time step with Eq. 6. In this case, 3- $\sigma$ bounds of the Monte carlo estimate (due to the use of finite number of samples) is also computed. The resultant CDFs are indicated as "Actual" in Fig. 3 ,

Note that both the above methods are sampling-based; while the former uses the same process noise values at all time steps, the latter selects a random sample of the process noise in each time step. The comparison between the above two is shown in Fig. 3, and it is seen that the proposed equivalent time-invariant approach compares well with that obtained using the true process noise. Thus, the use of time-invariant process noise has been validated, and this can now be used in inverse-FORM for quantifying the uncertainty in RUL.

\section{Quantification of Uncertainty in RUL}

Now that the proposed likelihood-based method has been verified, the next task is to replace Monte Carlo sampling with inverse-FORM. This is now easy because the original inverse-FORM can be used, since all the implicit relations have been eliminated. The different sources of uncertainty considered in this example are described below:

1. State Uncertainty: Typically, the state estimation, which is an inverse problem, is addressed using a filtering technique which can continuously estimate the uncertainty in the state when measurements are continuously available as a function of time. In this paper, the state estimation is not explicitly carried out. The state values are assumed to be available, and the uncertainty in the states are predetermined based on the authors' past experiences with the use of filtering techniques for the above described problem. There are two state variables (charge in the battery and charge associated with the capacitance) in this example and at any time instant, they are assumed to be normally distributed with a specified mean; for example, the mean of the initial states are set as $\left[4.14 \times 10^{4}, 0\right]$. For the purpose of illustration, three different values of $\mathrm{CoV}$ (Coefficient of variation, defined as the ratio between standard deviation and mean) are considered - 0.01, 0.02, 0.03 - and the analysis is repeated for each $\mathrm{CoV}$ value.

2. Loading Uncertainty: In this example, a constant amplitude loading is considered for the purpose of illustration. The amplitude is considered to be normally distributed $(N(1.375,1 / 6))$, and this distribution is truncated at a specified lower bound (0.75) and an upper bound (2.00).

3. Process Noise: In each time step, the two process noise values are assumed to normally distributed as $N(0,1)$ and $N(0,0.1)$ respectively.

The proposed inverse-FORM approach (including time-invariant process noise) is used to quantify the uncertainty in RUL and calculate the entire CDF of RUL (for each CoV corresponding to state uncertainty) continuously as a function of time. The $98 \%$ probability bounds of RUL for three cases (CoV of state uncertainty $=1 \%, 2 \%, 3 \%$ ) are shown in Figs. 4a $4 \mathrm{c}$ 


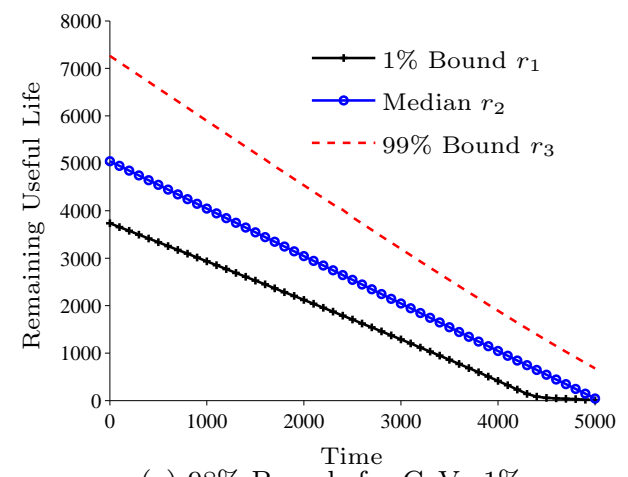

(a) $98 \%$ Bounds for $\mathrm{CoV}=1 \%$

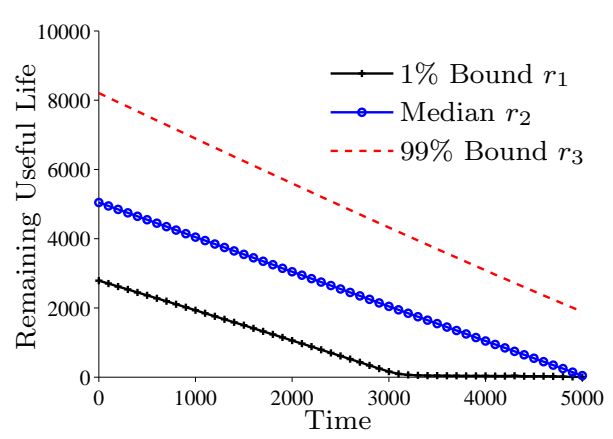

(c) $98 \%$ Bounds for $\mathrm{CoV}=3 \%$

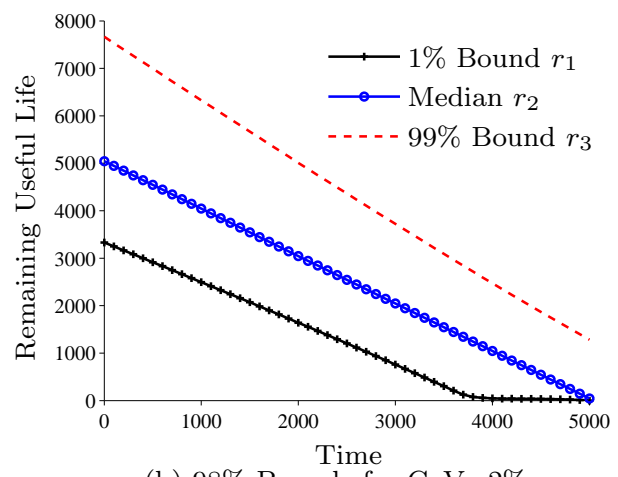

(b) $98 \%$ Bounds for $\mathrm{CoV}=2 \%$

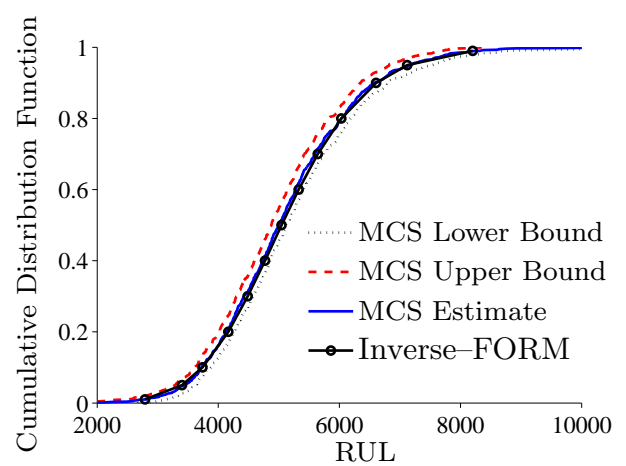

(d) Entire $\mathrm{CDF}$ for $T_{P}=0$ and $\mathrm{CoV}=3 \%$

Figure 4: Probability Distribution of RUL

The results from inverse-FORM also seem to be comparable against the Monte Carlo solution; for example, the CDFs from the proposed approach and the Monte Carlo simulation approach for RUL calculation at initial time and 3\% $\mathrm{CoV}$ are shown in Fig. 4d. In fact, a similar agreement was confirmed at multiple time instants for all the three $\mathrm{CoV}$ values, thereby verifying the proposed approach.

\section{Conclusion}

This paper discussed the use of the inverse first-order reliability method (inverse FORM) in order to compute the remaining useful life of aerospace components. The computation of remaining useful life is important in the context of component-level and system-level prognosis, and hence directly useful for online operations and decision-making. Conventionally, sampling-based algorithms have been used for quantifying the uncertainty in prognostic calculations, and may require several thousands of system evaluations in order to quantify the uncertainty in remaining useful life (RUL) with reasonable accuracy. On the contrary, the inverse FORM approach is an analytical algorithm and computes the uncertainty using a few system evaluations, and therefore is suitable for online prognosis. Further, the inverse FORM approach is invariant on repetition (as against sampling methods like Monte Carlo analysis), and hence may be preferred for system validation and certification purposes.

While this paper addressed a few challenges involved in using inverse-FORM for prognostics and calculating the uncertainty in RUL prediction, there are several other issues that need to be addressed. Practical systems are commonly subjected to different types of variable amplitude loading profiles such as block loading, Markov processes, general random processes, etc., and therefore, the proposed methods for uncertainty quantification need to be extended to consider variable amplitude loading. The assumption of constant amplitude loading implies that loading uncertainty is described using a single random variable, whereas variable amplitude loading profiles need to be described using multiple random variables, which not only increases the dimensionality of the problem, but also affects the uncertainty in the RUL prediction. Therefore, the impact of including variable amplitude loading on the uncertainty bounds of the RUL prediction needs to 
be investigated. Further, sensitivity analysis needs to be performed so that the contributions of the different sources of uncertainty to the overall uncertainty in RUL can be quantified. This paper did not consider the effect of model form uncertainty on prognosis; future research needs to quantify model form uncertainty and develop a method to rigorously account for model uncertainty in prognosis and RUL calculations. Finally, it is also necessary to quantify the robustness of the proposed approach, and thereby investigate the applicability of the methodology to practical engineering systems.

\section{Acknowledgments}

The work reported herein was in part funded by NASA ARMD/AvSafe project SSAT and by NASA OCT/AES project ACLO. The authors would also like to thank Dr. Matthew Daigle at NASA Ames Research Center for providing the model for the lithium-ion battery.

\section{References}

\footnotetext{
${ }^{1}$ Daigle, M., Foygel, M., and Smelyanskiy, V., "Model-based diagnostics for propellant loading systems," Aerospace Conference, 2011 IEEE, IEEE, 2011, pp. 1-11.

${ }^{2}$ Luo, J., Pattipati, K., Qiao, L., and Chigusa, S., "Model-based prognostic techniques applied to a suspension system," Systems, Man and Cybernetics, Part A: Systems and Humans, IEEE Transactions on, Vol. 38, No. 5, 2008, pp. 1156-1168.

${ }^{3}$ Goebel, K., Saha, B., and Saxena, A., "A comparison of three data-driven techniques for prognostics," 62nd Meeting of the Society For Machinery Failure Prevention Technology (MFPT), 2008, pp. 119-131.

${ }^{4}$ Sankararaman, S. and Mahadevan, S., "Uncertainty quantification in structural damage diagnosis," Structural Control and Health Monitoring, Vol. 18, No. 8, 2011, pp. 807-824.

${ }^{5}$ Sankararaman, S. and Mahadevan, S., "Bayesian methodology for diagnosis uncertainty quantification and health monitoring," Structural Control and Health Monitoring, Vol. In Press, 2011.

${ }^{6}$ Sankararaman, S., Ling, Y., Shantz, C., and Mahadevan, S., "Uncertainty quantification in fatigue damage prognosis," Annual Conference of the Prognostics and Health Management Society, 2009.

${ }^{7}$ Sankararaman, S., Ling, Y., Shantz, C., and Mahadevan, S., "Uncertainty quantification in fatigue crack growth prognosis," International Journal of Prognostics and Health Management, Vol. 2, No. 1, 2011.

${ }^{8}$ Farrar, C. and Lieven, N., "Damage prognosis: the future of structural health monitoring," Philosophical Transactions of the Royal Society A: Mathematical, Physical and Engineering Sciences, Vol. 365, No. 1851, 2007, pp. 623-632.

${ }^{9}$ Saha, B. and Goebel, K., "Uncertainty management for diagnostics and prognostics of batteries using Bayesian techniques," Aerospace Conference, 2008 IEEE, IEEE, 2008, pp. 1-8.

${ }^{10}$ Saha, B., Quach, C., and Goebel, K., "Optimizing battery life for electric UAVs using a Bayesian framework," Aerospace Conference, 2012 IEEE, IEEE, 2012, pp. 1-7.

${ }^{11}$ Ling, Y., Shantz, C., Mahadevan, S., and Sankararaman, S., "Stochastic prediction of fatigue loading using real-time monitoring data," International Journal of Fatigue, Vol. 33, No. 7, 2011, pp. 868 - 879.

${ }^{12}$ Ciampoli, M., "Time dependent reliability of structural systems subject to deterioration," Computers 83 structures, Vol. 67, No. 1-3, 1998, pp. 29-35.

${ }^{13}$ Liu, Y. and Mahadevan, S., "Efficient methods for time-dependent fatigue reliability analysis," AIAA journal, Vol. 47, No. 3, 2009, pp. 494-504.

${ }^{14}$ Der Kiureghian, A., Zhang, Y., and Li, C., "Inverse reliability problem," Journal of engineering mechanics, Vol. 120, 1994, pp. 1154.

${ }^{15}$ Haldar, A. and Mahadevan, S., Reliability assessment using stochastic finite element analysis, New York: John Wiley \& Sons, 2000.

${ }^{16} \mathrm{Li}$, H. and Foschi, R., "An inverse reliability method and its application," Structural Safety, Vol. 20, No. 3, 1998, pp. 257-270.

${ }^{17}$ Der Kiureghian, A., "The geometry of random vibrations and solutions by FORM and SORM," Probabilistic Engineering Mechanics, Vol. 15, No. 1, 2000, pp. 81-90.

${ }^{18}$ Sankararaman, S. and Mahadevan, S., "Likelihood-based representation of epistemic uncertainty due to sparse point data and/or interval data," Reliability Engineering ES System Safety, Vol. 96, No. 7, 2011, pp. 814 - 824.

${ }^{19}$ Balaban, E., Narasimhan, S., Daigle, M., Celaya, J., Roychoudhury, I., Saha, B., Saha, S., and Goebel, K., "A Mobile Robot Testbed for Prognostics-Enabled Autonomous Decision Making," Annual Conference of the Prognostics and Health Management Society, 2011.

${ }^{20}$ Chen, M. and Rincon-Mora, G. A., "Accurate electrical battery model capable of predicting runtime and I-V performance," IEEE Transactions on Energy Conversion, Vol. 21, No. 2, jun 2006, pp. $504-511$.

${ }^{21}$ Daigle, M., Saxena, A., and Goebel, K., "An Efficient Deterministic Approach to Model-based Prediction Uncertainty Estimation," Annual Conference of the Prognostics and Health Management Society, 2012.

${ }^{22}$ Sankararaman, S., Daigle, M., Saxena, A., and Goebel, G., "Analytical Algorithms to Quantify the Uncertainty in Remaining Useful Life Prediction," Aerospace Conference, 2013 IEEE, IEEE, Big Sky, MT, Mar 2013.
} 